\title{
Analytic model for the ballistic adsorption of polydisperse mixtures
}

\author{
Romualdo Pastor-Satorras* \\ Department of Earth, Atmospheric, and Planetary Sciences, Massachusetts Institute of Technology, Cambridge, Massachusetts 02139
}

(Received 20 October 1998)

\begin{abstract}
We study the ballistic adsorption of a polydisperse mixture of spheres onto a line. Within a mean-field approximation, the problem can be analytically solved by means of a kinetic equation for the gap distribution. In the mean-field approach, the adsorbed substrate is replaced by a set of effective particles having the same size, equal to the average diameter of the spheres in the original mixture. The analytic solution in the case of binary mixtures agrees quantitatively with direct Monte Carlo simulations of the model, and qualitatively with previous simulations of a related model in $d=2$. [S1063-651X(99)05705-0]
\end{abstract}

PACS number(s): 68.45.Da, 81.15.-z, 82.70.Dd, 68.10.Jy

\section{INTRODUCTION}

The adsorption of colloidal particles onto a surface is a subject of considerable interest due to its many practical applications in fields as diverse as physics, chemistry, biophysics, medicine, etc. [1]. Several models have been proposed so far, in an attempt to understand the physical properties of the adsorbed phase. In the random sequential adsorption (RSA) model [2-6], the adsorbing particles are located at random positions on the surface. If an incoming particle overlaps a previously adsorbed one, it is rejected; otherwise, it becomes irreversibly adsorbed. The RSA model does not consider the transport of the particles, and focuses only on the excluded volume effects. It is thus a valid approximation when the particles arrive at the surface purely by diffusion [5]. In the ballistic model (BM) [7-9], when an incoming particle fails to reach the surface directly, it is allowed to roll down over the previously adsorbed ones, following the direction of the steepest descent, until it reaches an equilibrium position. Particles that eventually rest on the surface are irreversibly adsorbed; otherwise, they are rejected. The BM is therefore a good approximation to describe adsorption in the presence of strong interactions, attracting the particles towards the surface [10-12].

In their original formulation, the aforementioned models, as well as their main variations, consider essentially the adsorption of a monodisperse suspension, in which the adsorbing particles all have the same size. Real-life suspensions, however, always possess an unavoidable degree of polydispersity. For instance, in some experimental situations the standard deviation of the particle size distribution may be up to $5-10 \%$ of the mean particle size $[13,14]$. Under such conditions, the effects of polydispersity may be indeed important.

The role of polydispersity has been studied in some detail in the RSA model. Theoretical works have dealt with binary mixtures of particles with greatly differing diameters [15], power-law size distributions [16,17], or general continuous size distributions [18]. Numerical simulations, on the other hand, have been performed in a wider variety of conditions:

\footnotetext{
*Present address: The Abdus Salam ICTP, P.O. Box 586, 34100 Trieste, Italy. Electronic address: romu@ictp.trieste.it
}

binary mixtures [19,20], uniform size distributions [19], Gaussian distributions [14,19], power-law distributions [17], etc.

In the context of the BM, it is worth noting the work of Senger et al. [10], where the authors describe a Monte Carlo model for the adsorption of a two-component mixture of hard spheres onto a plane, where the particles are under the simultaneous influence of diffusion and gravity. This is indeed a mixed model, which reproduces the standard RSA model in the limit of small particles, and the BM for large particles. The results reported by Senger et al., for particles large enough to be well inside the BM regime, are qualitatively similar to those found for the RSA of binary mixtures [19]: The maximum fraction of surface covered by the adsorbed particles-the jamming limit $\theta_{\infty}$-increases monotonically with the concentration $p$ of large particles, with a maximum in the limit $p \rightarrow 1^{-}$(i.e., $1-p$ arbitrarily small, but nonzero). For $p=0$ and $p=1$, the coverage corresponding to monodisperse adsorption is recovered.

In this paper we present an analytic model for the ballistic adsorption of mixtures of spherical particles with different diameters. The model can be solved in a mean field approximation by studying the kinetics of the gap density function [8]. Within this approach, we are able to derive a generic equation for an effective gap distribution. To test our equation, we solve it explicitly in the simplest case of a binary mixture. The analytic results obtained for the density at jamming $\theta_{\infty}$ match the findings of direct Monte Carlo simulations of the model. Moreover, the qualitative behavior of $\theta_{\infty}$ predicted by our model is the same as that reported by Senger et al. [10].

\section{MODEL}

Our model considers the adsorption onto a line of a polydisperse mixture whose degree of polydispersity is characterized, in general, by a continuous distribution of sizes $\rho(\sigma)$. The quantity $\rho(\sigma) d \sigma$ is defined as the fraction (bulk concentration in the infinite reservoir from which the particles are drawn) of spheres with diameter between $\sigma$ and $\sigma+d \sigma$. We assume $\rho$ to be normalized to 1 . Thus, for a monocomponent solution of particles of size $\sigma_{0}$, we have $\rho(\sigma)=\delta\left(\sigma-\sigma_{0}\right)$. The particles arrive at the line at rates $k(\sigma)$ per unit length per unit time. Assuming that the adsorbed substrate interacts 
a)

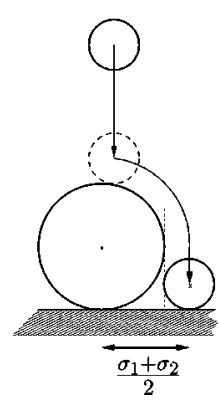

b)

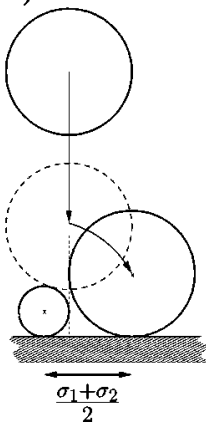

c)

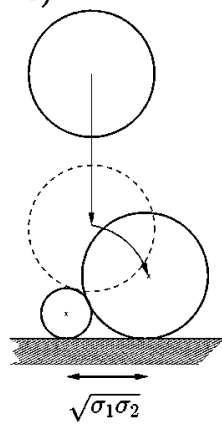

FIG. 1. Landing configurations for particles of different sizes $\sigma_{1}$ and $\sigma_{2}>\sigma_{1}$.

with the incoming particles only through excluded volume effects, we can select the appropriate units of time and set $k(\sigma) \equiv \rho(\sigma)$. Under these conditions, the problem translates into the sequential adsorption of particles of size $\sigma$, selected with a probability density $\rho(\sigma)$.

When an incoming particle lands on a preadsorbed one of exactly the same size, the adsorption rules are identical to the standard BM [8]. Figure 1 depicts the possible configurations involving particles of different diameters $\sigma_{1}$ and $\sigma_{2}$, with $\sigma_{1}<\sigma_{2}$. When a small particle rolls over a large one, the former finally falls on the surface and, after it is adsorbed, the centers of both particles are separated a horizontal distance $\Delta=\left(\sigma_{1}+\sigma_{2}\right) / 2$; Fig. 1(a) represents this case. When a large particle rolls over a small one, the rule adopted in our model is the one represented in Fig. 1(b), in which, after rolling, the centers of the two particles are also separated a distance $\Delta$.

The adoption of the rule pictured in Fig. 1(b) represents a major simplification of the model. It could be possible to argue that, in a more realistic treatment, the final configuration involving a large particle rolling over a small one should be the one depicted in Fig. 1(c). The surfaces of the particles are tangent after adsorption in this case, and their centers are separated a horizontal distance $\Delta_{R}=\sqrt{\sigma_{1} \sigma_{2}}$. Both rules can be easily implemented in a numerical simulation. However, the prescription 1(c) imposes an essential asymmetry among particles of different sizes. First of all, in our model, as defined by rules $1(\mathrm{a})$ and $1(\mathrm{~b})$, the final result of an adsorption event involving two spheres of different diameters is independent of the order in which the particles reach the surface. As a consequence, our model does not allow for "overhangs"; this means that, if $n(\sigma)$ is the density of adsorbed particles of size $\sigma$, then the fraction of covered surface $\theta$ is simply given by $\theta=\int d \sigma \sigma n(\sigma)$. This simple expression obviously does not hold in a model defined with the rule 1(c). These "Abelian" properties are eventually responsible for our model being analytically tractable.

\section{GENERAL MEAN-FIELD EQUATION}

The model defined in the preceding section can be analyzed by studying the density function of gaps-holes between two consecutive adsorbed particles. Let us define $G(x, t) d x$ as the number of gaps with a length between $x$ and $x+d x$ present at time $t$, per unit length of substrate. The time evolution of $G$ is obtained as a balance equation for the cre- ation and destruction of gaps caused by a single adsorption event [8]. Given $G$, the fraction of covered surface is defined by

$$
\theta(t)=1-\int_{0}^{\infty} x G(x, t) d x
$$

and, from here, we obtain the jamming limit as $\theta_{\infty}$ $=\lim _{t \rightarrow \infty} \theta(t)$.

In the case of the ballistic adsorption of a monodisperse solution of spheres of diameter $\sigma_{0}$, the equations for the density of gaps are [8]

$$
\begin{aligned}
\frac{\partial G(x, t)}{\partial t}= & -\left(x+\sigma_{0}\right) G(x, t)+2 \sigma_{0} G\left(x+\sigma_{0}, t\right) \\
& +2 \int_{x+\sigma_{0}}^{\infty} G(y, t) d y \text { for } x>\sigma_{0}, \\
\frac{\partial G(x, t)}{\partial t}= & 2 \sigma_{0} G\left(x+\sigma_{0}, t\right)+2 \int_{x+\sigma_{0}}^{\infty} G(y, t) d y \\
& \text { for } x<\sigma_{0} .
\end{aligned}
$$

The solution of Eqs. (2) and (3) is

$$
\begin{aligned}
G(x, t)= & e^{-\left(x+\sigma_{0}\right) t} t^{2} F\left(\sigma_{0} t\right) \exp \left\{2\left(1-e^{-\sigma_{0} t}\right)\right\} \\
& \text { for } x>\sigma_{0} ; \\
G(x, t)= & 2 \int_{0}^{t} d u u\left(1+\sigma_{0} u\right) e^{-\left(x+2 \sigma_{0}\right) u} F\left(\sigma_{0} u\right) \\
& \times \exp \left\{2\left(1-e^{-\sigma_{0} u}\right)\right\} \quad \text { for } x<\sigma_{0},
\end{aligned}
$$

where we have defined the auxiliary function

$$
F(t)=\exp \left\{-2 \int_{0}^{t} \frac{1-e^{-z}}{z} d z\right\} .
$$

For a polydisperse mixture, the naive application of this approach becomes considerably more involved. After a moment's reflection, it is easy to realize that, in this case, the final configuration resulting from an adsorption event taking place on a given gap depends on the sizes of the particles defining the boundaries of that gap. We should accordingly deal with a continuous set of functions $G_{\sigma^{\prime}, \sigma^{\prime \prime}}(x, t)$, defined as the densities of gaps created between particles of size $\sigma^{\prime}$ and $\sigma^{\prime \prime}$, for $\sigma^{\prime}, \sigma^{\prime \prime} \in[0, \infty]$. An enumeration of all the possible events occurring when adsorbing spheres of size $\sigma$ at rate $\rho(\sigma)$, would lead to a system of exact coupled integrodifferential equations for the magnitudes $G_{\sigma^{\prime}, \sigma^{\prime \prime}}$ that would completely determine the dynamics of the process. The magnitude of this task, especially when dealing with continuous size distributions $\rho(\sigma)$, seems to preclude any chance for an exact solution.

Fortunately, however, a great deal of insight can be gained by seeking a mean-field type of solution, based on the following argument: When the particles are free in the suspension, they are distinguishable and interact differently with the adsorbed phase, depending on their size. However, once they have been adsorbed, we can assume that they be- 
come indistinguishable, in the sense that the adsorbed particles interact with the incoming particles as if the former were all equal, with the same average diameter $\bar{\sigma}$ $=\int \sigma \rho(\sigma) d \sigma$. In other words, we can approximate the adsorbed phase with a set of effective particles with the same size $\bar{\sigma}$, interacting with incoming particles of size $\sigma$. Assuming this simplification, we need only a single effective gap distribution $G$, defined by the gaps bounded by the adsorbed effective particles.

We note the important fact that the aforementioned meanfield approximation does not imply at all that the density of adsorbed particles is proportional to the bulk density, $n(\sigma)$ $\propto \rho(\sigma)$. This relation, which can be true at the first stages of the adsorption process, does not hold close to the jammed state. This last statement is most easily seen in binary mixtures (see Sec. IV).

The kinetic equation for the effective gap density can be written in the generic form

$$
\begin{aligned}
\frac{\partial G(x, t)}{\partial t}= & -\int_{0}^{x} d \sigma \rho(\sigma)(x+\bar{\sigma}) G(x, t) \\
& +2 \int_{0}^{\infty} d \sigma \rho(\sigma)\left(\frac{\sigma}{2}+\frac{\bar{\sigma}}{2}\right) G(x+\sigma, t) \\
& +2 \int_{0}^{\infty} d \sigma \rho(\sigma) \int_{x+\sigma}^{\infty} d y G(y, t) .
\end{aligned}
$$

The origin of the different terms in Eq. (5) is the following: The destruction of gaps of length $x$ is due to the landing of a particle of size $\sigma$ on any point of an interval of length $x$ $+\bar{\sigma}$ centered on the gap. After averaging over the distribution of incoming particles of size $\sigma<x$, we obtain the first term in Eq. (5). A gap of length $x$ can be created by the impact of particles of size $\sigma$ on either of the particles of effective size $\bar{\sigma}$ defining a gap of length $x+\sigma$. These events, which happen at rate $\rho(\sigma)$, account for the second term in Eq. (5). The last term is due to the averaged creation of gaps of length $x$ by direct deposition of a particle of size $\sigma$ onto a gap of length $y>x+\sigma$. We remark again that Eq. (5) owes its relatively simple form to the choice of the "Abelian" rule 1(b) in the definition of the model. A much more complex expression would have been obtained with rule 1(c).

Equation (5) can be expressed in a more compact way by integrating by parts its last term. Defining the distribution function $\Psi(x)=\int_{0}^{x} \rho(\sigma) d \sigma$, we obtain

$$
\begin{aligned}
\frac{\partial G(x, t)}{\partial t}= & -\Psi(x)(x+\bar{\sigma}) G(x, t) \\
& +\int_{0}^{\infty} d \sigma[\rho(\sigma)(\sigma+\bar{\sigma})+2 \Psi(\sigma)] G(x+\sigma, t) .
\end{aligned}
$$

Equation (6) is the final expression of the mean-field theory for our model of polydisperse ballistic adsorption. As a consistency check, we consider the trivial scenario of a monodisperse suspension. In this case, by setting $\rho(\sigma)$ $=\delta\left(\sigma-\sigma_{0}\right)$ and $\Psi(x)=\Theta\left(x-\sigma_{0}\right)$, where $\Theta$ is the Heavi- side step function, we immediately recover the equations for a single-size distribution, as given by Eqs. (2) and (3).

\section{BINARY MIXTURES}

In order to test the validity of our mean-field theory, we now proceed to solve explicitly Eq. (6) in the case of a binary mixture, composed of particles of size $\sigma_{1}=1$, which adsorb onto the surface at rate $\phi_{1}$, and particles of size $\sigma_{2}$ $=r>1$, adsorbing at rate $\phi_{r}=1-\phi_{1}$. As an aside, in this simple setting we can estimate the variations in the jamming limit due to the adoption of rule 1(b) instead of 1(c). One can expect that, for small values of $r$, the outcome of both models should be similar. Indeed, numerical simulations show that, for values of $r<2$, the difference between prescriptions is always less than $1 \%$, for all values of $\phi_{r}$.

The density function for a binary mixture has the form $\rho(\sigma)=\phi_{1} \delta(\sigma-1)+\phi_{r} \delta(\sigma-r)$, whereas the distribution function is $\Psi(x)=\phi_{1} \Theta(x-1) \Theta(r-x)+\Theta(x-r)$, and the average size $\bar{\sigma}=\phi_{1}+r \phi_{r}$. By inserting these expressions into Eq. (5) or (6), we obtain the following set of equations:

$$
\begin{aligned}
\frac{\partial G(x, t)}{\partial t}= & -(x+\bar{\sigma}) G(x, t)+\phi_{1}(1+\bar{\sigma}) G(x+1, t) \\
& +\phi_{r}(r+\bar{\sigma}) G(x+r, t)+2 \phi_{1} \int_{x+1}^{\infty} G(y, t) d y \\
& +2 \phi_{r} \int_{x+r}^{\infty} G(y, t) d y \quad \text { for } x>r \\
\frac{\partial G(x, t)}{\partial t}= & -\phi_{1}(x+\bar{\sigma}) G(x, t)+\phi_{1}(1+\bar{\sigma}) G(x+1, t) \\
& +\phi_{r}(r+\bar{\sigma}) G(x+r, t)+2 \phi_{1} \int_{x+1}^{\infty} G(y, t) d y \\
& +2 \phi_{r} \int_{x+r}^{\infty} G(y, t) d y \quad \text { for } 1<x<r \\
& +2 \phi_{r} \int_{x+r}^{\infty} G(y, t) d y \quad \text { for } 0<x<1 . \\
& +\phi_{r}(r+\bar{\sigma}) G(x+r, t)+2 \phi_{1} \int_{x+1}^{\infty} G(y, t) d y \\
& \phi_{1}(1+\bar{\sigma}) G(x+1, t) \\
& \\
&
\end{aligned}
$$

We observe that, for a binary mixture, one could in principle try to solve the model exactly by determining the rate equations for the densities of gaps delimited by particles of size 1 and $r$, namely, $G_{1,1}, G_{r, r}$, and $G_{1, r}$. However, in this case one would end up with a set of nine coupled equations. The simplification achieved through the mean-field theory is evident here.

We consider in particular the case $1<r<2$. To solve the kinetic equations, we seek in Eq. (7) a solution of the form $G(x, t)=e^{-(x+\bar{\sigma}) t} H(t)$. With this substitution, we are led to the equation for $H(t)$ : 


$$
\frac{d \ln H}{d t}=\phi_{1}\left[(1+\bar{\sigma})+\frac{2}{t}\right] e^{-t}+\phi_{r}\left[(r+\bar{\sigma})+\frac{2}{t}\right] e^{-r t} .
$$

The solution of Eq. (10), with the initial condition $H(0)$ $=0$, is

$$
\begin{aligned}
H(t)= & t^{2} \exp \left\{\phi_{1}(1+\bar{\sigma})\left(1-e^{-t}\right)\right\} \\
& \times \exp \left\{\phi_{r}(r+\bar{\sigma})\left(1-e^{-r t}\right) / r\right\}[F(t)]^{\phi_{1}}[F(r t)]^{\phi_{r},}
\end{aligned}
$$

where $F(t)$ is defined in Eq. (4). Upon substituting this result into Eq. (8), we look for a solution of this equation of the form $G(x, t)=e^{-\phi_{1}(x+\bar{\sigma}) t} Q(x, t)$. The equation determining $Q$ is

$$
\frac{\partial Q(x, t)}{\partial t}=e^{-\phi_{r}(x+\bar{\sigma}) t} \frac{d H(t)}{d t},
$$

from which $Q(x, t)$ is obtained by direct integration, together with the initial condition $Q(x, 0)=0$ :

$$
Q(x, t)=e^{-\phi_{r}(x+\bar{\sigma}) t} H(t)+\phi_{r}(x+\bar{\sigma}) \int_{0}^{t} d u e^{-\phi_{r}(x+\bar{\sigma}) u} H(u) .
$$

Finally, by substituting the solutions of Eqs. (7) and (8) into the appropriate range of values of $x$ in Eq. (9) (and taking into account that $r<2$ ), we can directly integrate this equation. Using Eq. (1), and after performing some algebraic manipulations, we obtain the density of adsorbed particles as a function of time:

$$
\begin{aligned}
\theta(t)= & \int_{0}^{t} d u H(u) \mathcal{F}_{1}(u)+\phi_{r} \int_{0}^{t} d u H(u) \mathcal{F}_{2}\left(\phi_{1} t+\phi_{r} u\right) \\
& +\phi_{1} \phi_{r}(1+\bar{\sigma}) \int_{0}^{t} d u H(u) \int_{u}^{t} d v \mathcal{F}_{3}\left(\phi_{1} v+\phi_{r} u\right) \\
& +2 \phi_{1} \phi_{r} \int_{0}^{t} d u H(u) \int_{u}^{t} d v \mathcal{F}_{4}\left(\phi_{1} v+\phi_{r} u\right),
\end{aligned}
$$

where we have introduced the auxiliary functions

$$
\begin{gathered}
\mathcal{F}_{1}(z)=\frac{e^{-\bar{\sigma} z}}{z^{3}}\left\{\left[2 \phi_{r}+\left(1+\phi_{r}+\phi_{r} \bar{\sigma}\right) z+(\bar{\sigma}+1) z^{2}\right] e^{-z}\right. \\
\left.-\left[2 \phi_{r}+\phi_{r}(r+\bar{\sigma}) z\right] e^{-r z}\right\}, \\
\mathcal{F}_{2}(z)=\frac{e^{-\bar{\sigma} z}}{z^{3}}\left\{-\left[2+(\bar{\sigma}+2) z+(\bar{\sigma}+1) z^{2}\right] e^{-z}\right. \\
\left.+\left[2+(\bar{\sigma}+2 r) z+r(\bar{\sigma}+r) z^{2}\right] e^{-r z}\right\}, \\
\mathcal{F}_{3}(z)=\frac{e^{-\bar{\sigma} z}}{z^{3}}\left\{-[2+(\bar{\sigma}+1) z] e^{-z}+[2+(\bar{\sigma}+2 r-1) z\right. \\
\left.\left.+(r-1)(r+\bar{\sigma}) z^{2}\right] e^{-r z}\right\},
\end{gathered}
$$

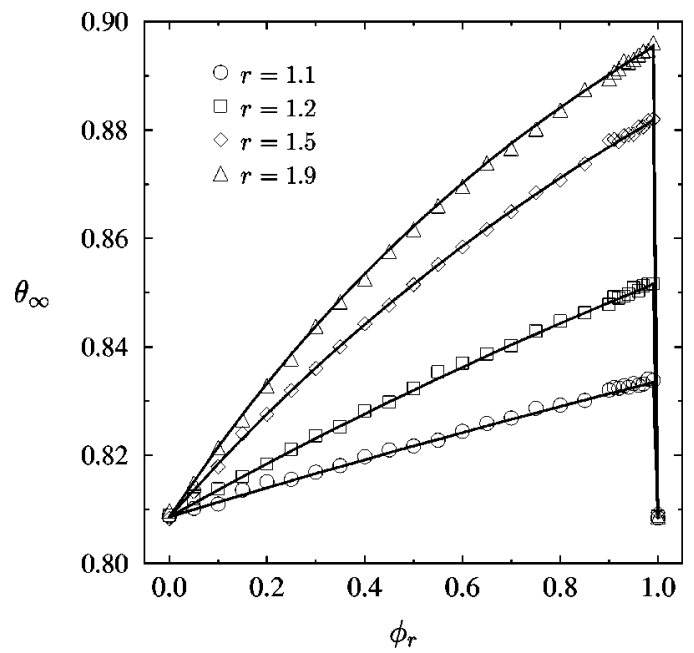

FIG. 2. Jamming limit as a function of the concentration fraction $\phi_{r}$ of large particles, for different values of the diameter ratio $r$. Comparison between numerical simulations (hollow symbols) and the mean-field prediction (full lines).

$$
\begin{aligned}
\mathcal{F}_{4}(z)= & \frac{e^{-\bar{\sigma} z}}{z^{4}}\left\{-[3+(\bar{\sigma}+1) z] e^{-z}\right. \\
& +\left[3+(\bar{\sigma}+3 r-2) z+\frac{1}{2}(r-1)(3 r+2 \bar{\sigma}-1) z^{2}\right. \\
& \left.\left.+\frac{1}{2}(r-1)^{2}(r+\bar{\sigma}) z^{3}\right] e^{-r z}\right\} .
\end{aligned}
$$

We can estimate the theoretical predictions of this meanfield solution by numerically integrating the previous expression in the limit $t \rightarrow \infty$. Figure 2 shows in full lines the results of the integration for different values of $r$. The symbols represent data obtained from direct Monte Carlo simulations of the model on a line of length 1000 with periodic boundary conditions. We observe that the predictions of the mean-field theory are in excellent agreement with the numerical simulations.

From Fig. 2 we conclude that, for $\phi_{r}<1$, the jamming limit is an increasing monotonic function of this variable. For $\phi_{r}=0$ or $\phi_{r}=1$ (only small or large particles, respectively), we recover, for any $r$, the prediction of the standard BM model, $\theta_{\infty}^{\mathrm{BM}} \simeq 0.808$ [8]. For small values of $\phi_{r}, \theta_{\infty}$ grows linearly, $\theta_{\infty} \simeq \theta_{\infty}^{\mathrm{BM}}+\alpha(r) \phi_{r}$, with a slope $\alpha(r)$ that increases with $r$. The value of the slope at the origin can be easily estimated by Taylor expanding the expression for $\theta(t)$. The jamming limit exhibits a maximum located at $\phi_{r}$ $\rightarrow 1^{-}$, in qualitative agreement with the findings of Senger et al. [10]. The actual value of the maximum $\theta_{\infty}^{\max }(r)$ is an increasing function of $r$, with an apparent tendency to saturate at large $r$. In the limit $\phi_{r} \rightarrow 1$, and for $r \gg 1$, we can estimate the limiting value of $\theta_{\infty}^{\max }(r)$ [19]: In this limit, the large particles cover first a fraction of surface $\theta_{\infty}^{\mathrm{BM}}$ of the line, leaving free a surface $1-\theta_{\infty}^{\mathrm{BM}}$ that is afterwards covered until jamming by the small particles. The total coverage is therefore bounded by $\theta_{\infty}^{\max }(r) \leqslant \theta_{\infty}^{\mathrm{BM}}+\left(1-\theta_{\infty}^{\mathrm{BM}}\right) \theta_{\infty}^{\mathrm{BM}}=\theta_{\infty}^{\mathrm{BM}}(2$ $\left.-\theta_{\infty}^{\mathrm{BM}}\right) \simeq 0.96339$. Monte Carlo simulations confirm this ex- 
treme, yielding the value $\theta_{\infty}^{\max }=0.964 \pm 0.001$ for $r=20$ and $\phi_{r}=0.99$.

\section{CONCLUSIONS}

To sum up, in this paper we have presented an extension of the classical ballistic model [7-9], describing the ballistic adsorption onto a line of a polydisperse mixture of spherical particles of different sizes $\sigma$, present with a bulk concentration $\rho(\sigma)$. The model is solved by means of a mean-field equation, which approximates the adsorbed phase by a set of effective particles all having the same average diameter $\bar{\sigma}$ $=\int d \sigma \sigma \rho(\sigma)$, interacting with incoming particles of variable size. To check our mean-field approximation, we have explicitly solved the case of a binary mixture. The perfect match of the theoretical solution and the numerical simula- tions confirms the validity of the mean-field approximation, at least for this particular case. Our findings agree also with numerical simulations of a related model in two dimensions [10]. On theoretical grounds, the proposed mean-field approach could be a first step toward dealing with more complex situations, such as, for example, higher dimensionalities, where the assumption of an effective layer of adsorbed particles would be more reasonable, or the case of adsorption onto a substrate initially covered with impurities.

\section{ACKNOWLEDGMENTS}

This work was financially supported by a grant from the Ministerio de Educación y Cultura (Spain). I would like to thank Professor M. Rubí for helpful discussions and Dr. M. C. Miguel for a careful reading of the manuscript.
[1] J. Evans, Rev. Mod. Phys. 65, 1281 (1993).

[2] A. Rényi, Sel. Trans. Math. Stat. Prob. 4, 203 (1963).

[3] J. Feder, J. Theor. Biol. 87, 237 (1980).

[4] P. Schaaf and H. Reiss, J. Chem. Phys. 92, 4824 (1988).

[5] B. Senger, J.-C. Voegel, P. Schaaf, A. Johner, A. Schmitt, and J. Talbot, Phys. Rev. A 44, 6926 (1991).

[6] J. J. Ramsden, Phys. Rev. Lett. 71, 295 (1993).

[7] P. Meakin and R. Jullien, J. Phys. (Paris) 48, 1651 (1987).

[8] J. Talbot and S. M. Ricci, Phys. Rev. Lett. 68, 958 (1992).

[9] A. P. Thompson and E. D. Glandt, Phys. Rev. A 46, 4639 (1992).

[10] B. Senger, R. Ezzeddine, F. J. Bafaluy, P. Schaaf, F. J. G. Cuisinier, and J.-C. Voegel, J. Theor. Biol. 163, 457 (1993).

[11] P. Schaaf, P. Wojtaszczyk, B. Senger, J. C. Voegel, and H. Reiss, Phys. Rev. E 51, 4292 (1995).
[12] R. Pastor-Satorras and J. M. Rubí, Phys. Rev. Lett. 80, 5373 (1998).

[13] P. Wojtaszczyk, P. Schaaf, B. Senger, M. Zembala, and J. C. Voegel, J. Chem. Phys. 99, 7198 (1993).

[14] Z. Adamczyk, B. Siwek, M. Zembala, and P. Weronsky, J. Colloid Interface Sci. 185, 236 (1997).

[15] J. Talbot and P. Schaaf, Phys. Rev. A 40, 422 (1989).

[16] P. L. Krapivsky, J. Stat. Phys. 69, 135 (1992).

[17] N. V. Brilliantov, Y. A. Andrienko, P. L. Krapivsky, and J. Kurths, Phys. Rev. Lett. 76, 4058 (1996).

[18] G. Tarjus and J. Talbot, J. Phys. A 24, L931 (1991).

[19] P. Meakin and R. Jullien, Phys. Rev. A 46, 2029 (1992).

[20] B. Bonnier, Y. Leroyer, and E. Pommiers, J. Phys. I 2, 379 (1992). 